\title{
A novel index modulation scheme with impedance matching
}

\author{
Hye Yeong Lee, Soo Young Shin \\ Department of IT Convergence Engineering, Kumoh National Institute of Technology, South Korea
}

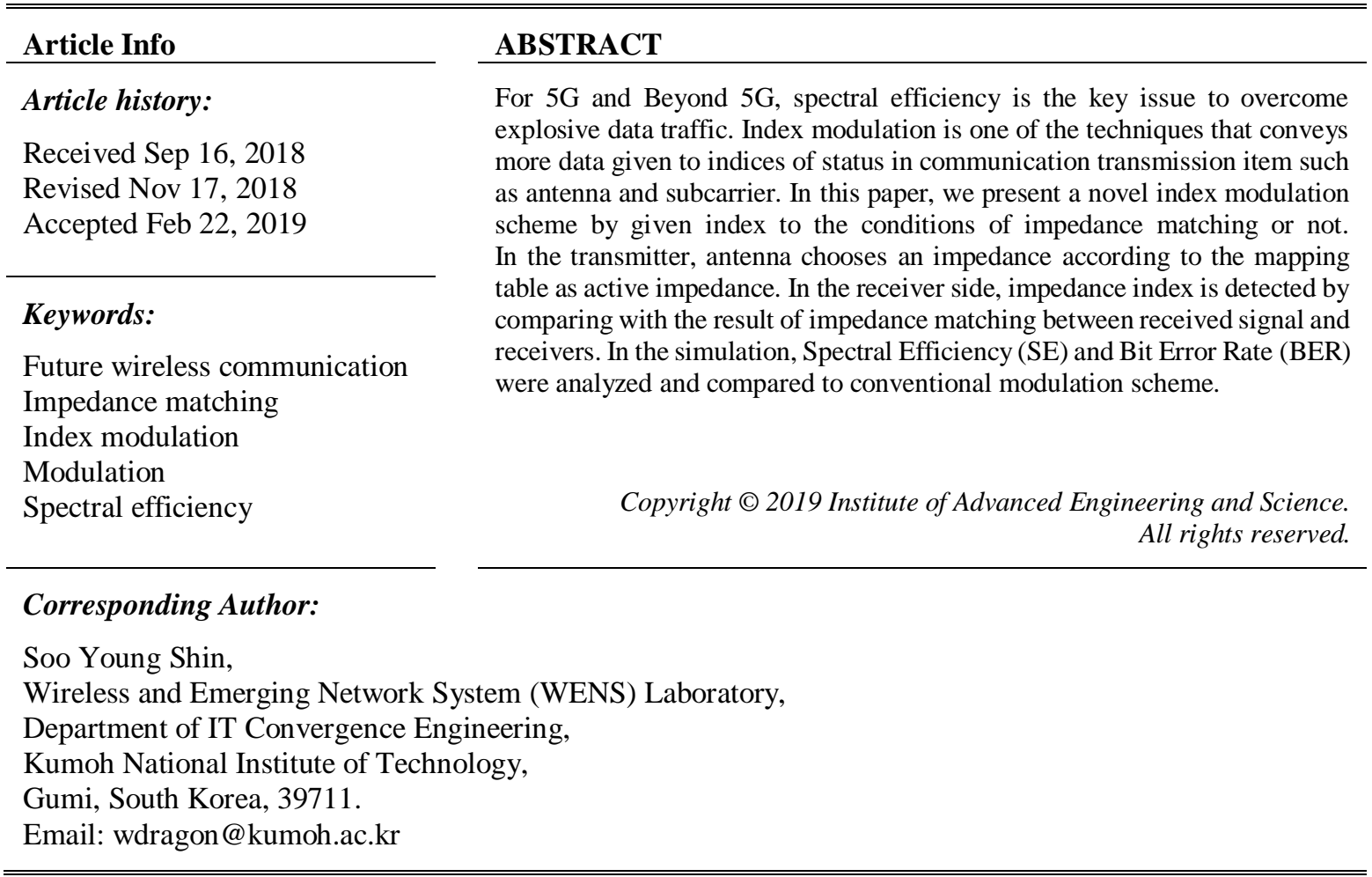

\section{INTRODUCTION}

Recently, future wireless network faced on the explosive increaing of data traffic [1]. According to Cisco's VNI Global Data Traffic Forecast, by 2020, wireless data traffic is expected to increase to five times the current level to49EB (Exabyte) [2]. In order to solve this increase, many researches are focusing on how to transmit a large amount of information in a communication channel [3]. Morover, frequency is the limited resource, so it is necessary to transmit data efficiently with limited frequency conditions. One of the way to improve spectral efficiency is modulation.

Conventionally, carrier modulation techniques have been researched by exploiting characteristic of carrier. The parameters of carrier including phase, amplittude and frequency are used one or more to modulate such as Phase-Shift Keying (PSK) and Quadrature Amplitude Modulation (QAM) which is modulated amplitude and phase at the same time [4],[5]. However, these modulation techniques can augment both error rate and design complexity of the system as the amount of information to be transmitted increment.

Index Modulation (IM) suggested to improve performance of spectral efficiency for 5G and Beyond 5G. The concept of IM is to transmit additional information by utilizing antennas, subcarriers and time slots [6], [7] etc. IM can reduce the complexity of design as well as improve spectral efficiency and energy efficiency in the system. Commonly, there are two IM scheme that a lot of reserach has been doing, one is Spatial Modulation (SM) [8] and the other is Orthogonal Frequency Division Multiplexing Index Modulation (OFDMIM) [9]. SM is spatial multiplexing MIMO scheme using antenna index in order to overcome Inter Channel Interference(ICI) and improve SE [10], [11]. In transmitter, data is transmitted using one antenna in multi array antenna according to the data bits. The data can be detected both actived antenna index and data symbol in receiver. OFDM-IM is a multi-carrier transmission scheme inspiring by the concept of SM [12], [13]. The key principle of the scheme is given indices to the group of subcarrier using On-Off Keying(OOK). That means the whole carrier is divided into small groups, and information is assigned to the activated 
subcarrier group by 1 and the other groups by 0 [14]. It can detect index of the activated subcarrier's group or not.

In this paper, we introduce a novel index modulation scheme Impedance Index Modulation, called IIM, by exploitling impedance matching. In general, when two circuits are connected with different impedance value, a reflection voltage is generated at the end of circuit, that is the impedance mismatch. If mismatching problem happens in the system, it is impossible to transmit maximum power, so it degrades the power efficiency [15]. To avoid this problem, impedance matching system is necessary to build between different impedances. Like this, impedance mismatch was considered to be a value to be removed from the system because of the power loss. However, in this paper, we propose a method to transmit data by using this removed value. The key point of IIM is that assigned binary1 to perfect match case and binary 0 is given mismatch case. When one impedance is selected in the transmitter, received signal is detected using comparison of impedance match or not in the receiver. The information is decoded from the mapping table using the detected impedance. Through this process, it is possible to transmit additional information by using the impedance matching index.

We describe the basic concept of impedance matching in Section 2. In Section 3, we show the proposed system divided two part, system model and impedance detecting algorithms. Section 4 contains simulation result about bit error rate and spectral efficiency. Finally, conclusions are given in Section 5.

\section{RELATED WORK}

At first, we introduce the basic principle of impedance matching scheme to apply our proposed system. Impedance matching is a technique, in which matches the impedance of the source and load to be equal, to transmit maximum power from source to load in the RF system[16]-[18]. This technique is essentially used for antenna and speaker design. Especially, it is an important factor in antenna design to maximize power efficiency of system by reducing power loss in RF.

Figure 1 shows a system model of impedance matching. As Shown in Figure 1(a), when source impedance $Z_{S}$ and load impedance $Z_{L}$ with different properties $\left(Z_{S} \neq Z_{L}\right)$ are connected, the reflection voltage $V_{\text {reflected }}$ is generated contact boundary. When the reflected voltage happens, the power loss of the signal occurs, moreover, the efficiency of the overall system is lowered. For this critical reason, the impedance matching network is widely used for adding between two impedances like Figure 1(b) [18].

When impedances match perfectly, there is no power loss, so the power of load impednace will be maximized. In impednace matching system, load impedance is $Z_{L}=R_{L}+j X_{L}$ and soure impedance is $Z_{S}=$ $R_{S}+j X_{S}$. The power of load impedance $P_{L}$ is defined as,

$$
P_{L}=\frac{1}{2} \frac{\left|V_{S}\right|^{2} R_{L}}{\left(R_{S}+R_{L}\right)^{2}+\left(X_{S}+X_{L}\right)^{2}}
$$

where $V_{S}$ is source voultage. Based on the (1), the load impedance is the same size as the source impedance and the opposite direction, then the load impedance power will be the maximum, that means $Z_{S}=Z_{L}{ }^{*}[19]$. Thus, the maximum power transfer theory is satisfied when the impedance is the same, at that time impedance is matched.

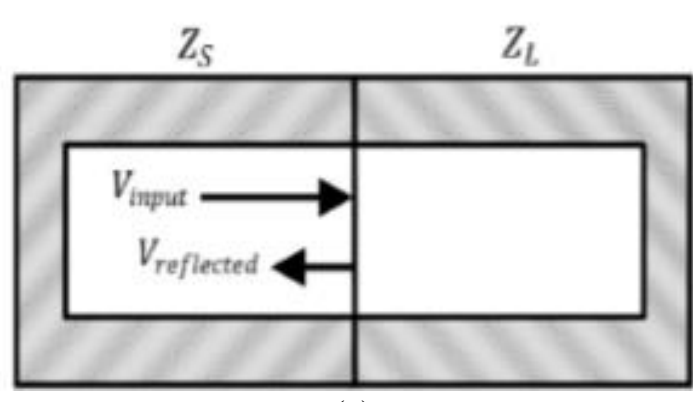

(a)

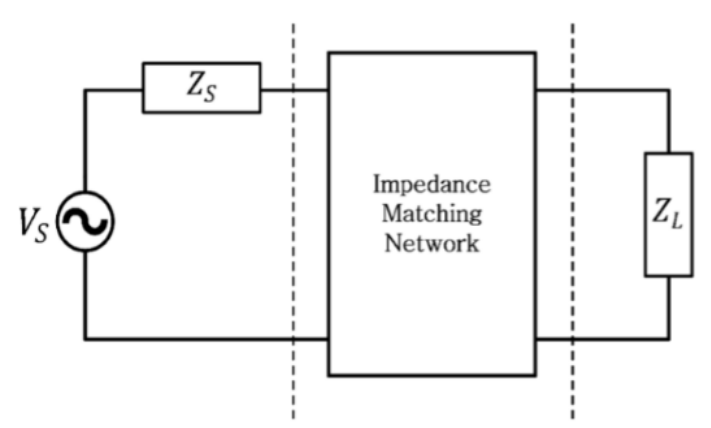

(b)

Figure 1. Effects of the impedance matching (a) reflection voltage, (b) system model 


\section{IMPEDANCE INDEX MODULATION}

\subsection{System Model}

In this paper, we suggest the impedance index modulation that is inspiring by the principle of impedance matching, in order to improve spectral efficiency. Let us suppose that the channel between the transmitter and receiver is a transmission line for applying impedance matching to system model.

Figure 2 shows the proposed system model. Each transmitter and receiver has $N_{Z}$ impedances $\left(Z_{1}, Z_{2}, \cdots, Z_{k}, k \in\left\{1, \cdots, N_{Z}\right\}\right)$. Through switch, one impedance is connected to the RF antenna in the transmitter. On the other hand, all impedance is connected to the RF antenna on the received side. The block diagram of the impedance index modulation is shown in Figure 3. First, when input bits $B$ come to system, it divided two group of bits like impdedance index $B_{1}=\log _{2}\left\lfloor N_{Z}\right\rfloor$ bits and conventional symbol mdoulation $B_{2}=\log _{2} M$ bits using bit splitter. $\mathrm{M}$ is modulation order. The impedance index is selected according to the mapping table. The selected impedance connects to antenna using switch, which means one impedance is chose in the transmitter. We assume that the speed of impedance switching in transmitter is not affect signal transmission. When the signal transmit through the channel, the received signal go through all of receiver impedance and check the result that impedance match or not. During impedance matching process, impedance index is detected. And then, symbol can be demodulated. We describe the part of the impedance index detection in detail in the next section.
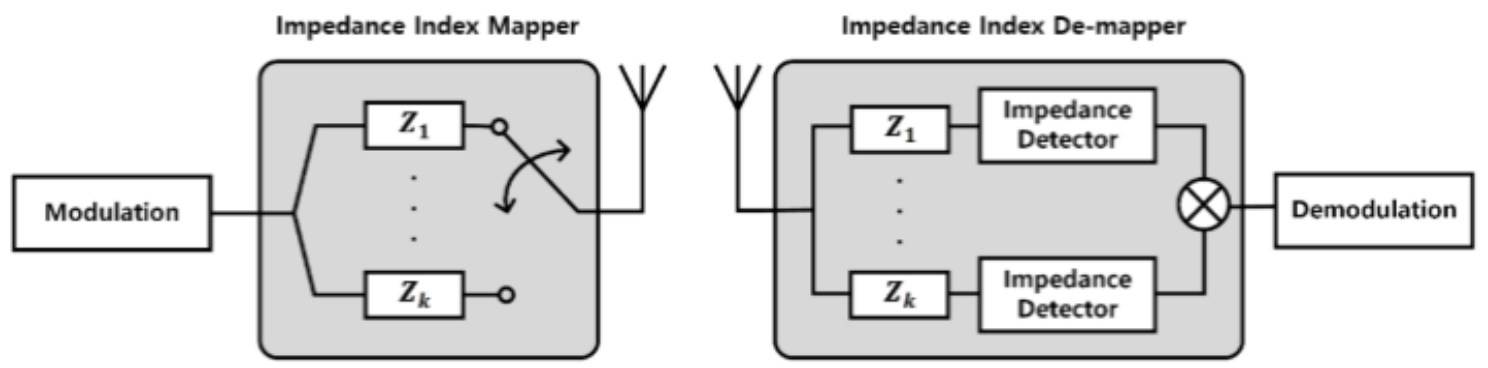

Figure 2. System model

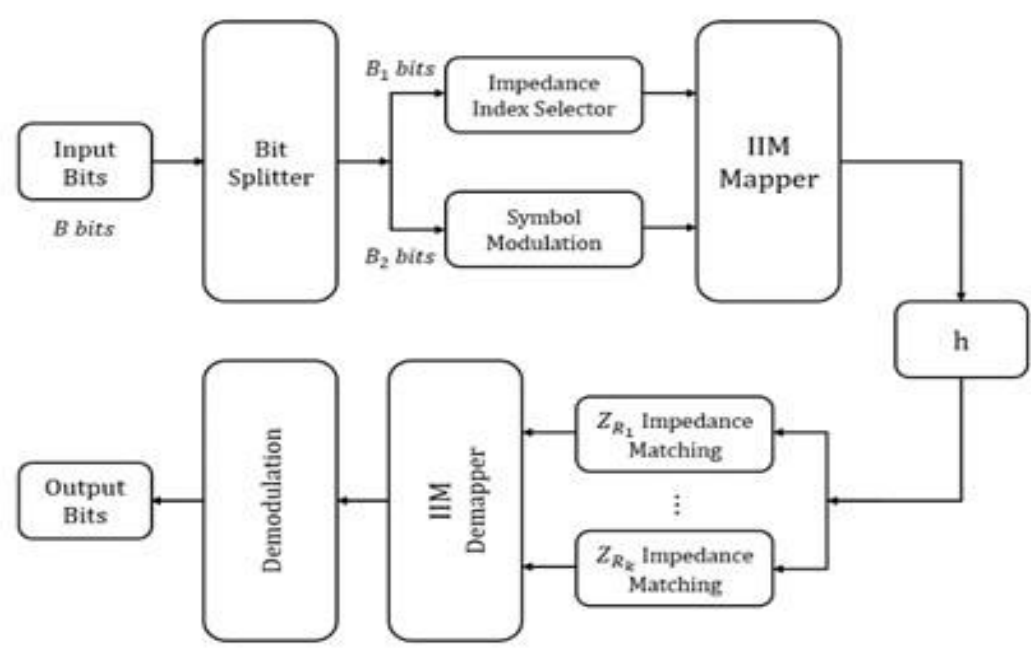

Figure 3. Block diagram of the impedance index modulation

Table 1 represent the mapping table of impedance index. The mapping table shows impedance index and symbol value according to the bits. Let us suppose the condition using four impedances and the conventional modulation scheme as BPSK. In this case, the inpedance index bit is $B_{1}=\log _{2}\lfloor 4\rfloor=2 \mathrm{bits}$ and symbol modulation bit is $B_{2}=\log _{2} 2=1 \mathrm{bits}$, so the total incoming bits is $3 \mathrm{bits}$. For example, when incoming bits are ' 010 ', $B_{1}$ is first two bits as ' 01 ' that means impedance index is 2 based on the mapping table, and $B_{2}$ is last one bit as ' 0 ' that said symbol data is -1 in BPSK system. 
Table 1. The Mapping Table in IIM $\left(N_{Z}=4\right.$ with BPSK)

\begin{tabular}{|c|c|c|c|}
\hline \multicolumn{2}{|c|}{ Bits } & \multirow{2}{*}{$\begin{array}{l}\text { Impedance } \\
\text { Index }\end{array}$} & \multirow{2}{*}{ Symbols } \\
\hline$B_{1}$ & $B_{2}$ & & \\
\hline 00 & 0 & 1 & -1 \\
\hline 00 & 1 & 1 & +1 \\
\hline 01 & 0 & 2 & -1 \\
\hline 01 & 1 & 2 & +1 \\
\hline 10 & 0 & 3 & -1 \\
\hline 10 & 1 & 3 & +1 \\
\hline 11 & 0 & 4 & -1 \\
\hline 11 & 1 & 4 & +1 \\
\hline
\end{tabular}

\subsection{Impedance Index Detecting Algorithm}

Figure 4 shows our proposed algorithme for detecting impedance index. The main idea of index detecting is to use maximum power transfer theorem based on the information of impedance match or not. In this section, we show how to apply the principle of the impedance match to the index modulation and detect to impedance index.

In the wireless communication system, the general received signal is defined as,

$$
\mathbf{y}=\sqrt{P_{T}} \boldsymbol{h} \boldsymbol{x}+\boldsymbol{n}
$$

Where $\boldsymbol{h}$ is channel, $\boldsymbol{n}$ is Additive White Gaussian Noise (AWGN) and $P_{T}$ is the power of transmitter.

In case of the impedance matching, the matching result affects to load power. By applying impedance match in the proposed system, the source impedance $Z_{S}$ is matched to the transmitter impedance $Z_{T}$ and the load impedance $Z_{L}$ will be the receiver impedance $Z_{R}$.

As shown Figures 2 and 3, when one impedance index is demodulated in the system, one selected transmit impedance is compared with all of the receiver impedances by using the received signal. Based on (1), the power of $k^{\text {th }}$ receiver impedance $P_{R_{k}}$ is described as

$$
P_{R_{k}}=\frac{1}{2} \frac{\left|V_{T}\right|^{2} Z_{R_{k}}}{\left(z_{T}+Z_{R_{k}}\right)^{2}}, k \in\left\{1, \cdots, N_{Z}\right\}
$$

Where $Z_{T}$ is the transmission impedance, $Z_{R_{k}}$ is $k^{\text {th }}$ impedance of receiver, and $V_{T}$ is the transmitter power. In this case, $V_{T}$ and $Z_{T}$ are variable value up to the received signal. Based on (3), $V_{T}$ can be omitted as it does not change if the receiver impedance changes. To use easily the principle of impedance match, we derived the attenuation coefficient and gave to the weight value for each result of the impedance matching process in the receiver. Therefore, the attenuation coefficient of the transmission impedance and the receiver impedance is derived from (3) and expressed as follows,

$$
\gamma_{k}=\frac{z_{R_{k}}}{\left(z_{T}+z_{R_{k}}\right)^{2}}
$$

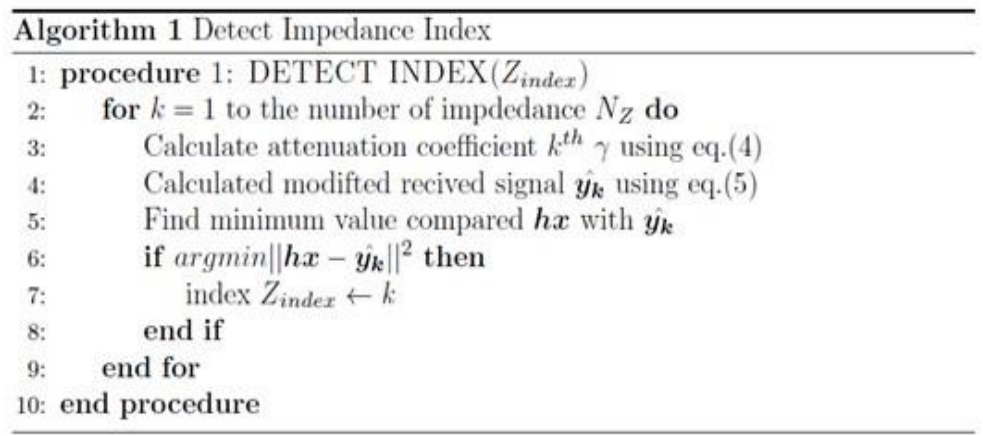

Figure 4. The algorithm of detecting impedance index 
It can be confirmed that the impedance of receiver power becomes maximum when the transmitter and receiver impedances are matched according to the attenuation coefficient. The received power value is determined according to the attenuation coefficient, which is expressed by multiplying the received signal. When one impedance index is restored, comparison is made by the number of impedance in the receiver. In one process, the received signal by exploiting the attenuation coefficient can be expressed as (5)

$$
\begin{aligned}
& \widehat{\boldsymbol{y}}_{k}=\gamma_{k} \mathbf{y} \\
& Z_{\text {index }}=\arg \min _{k}\left\|\boldsymbol{h} \boldsymbol{x}-\widehat{\boldsymbol{y}}_{k}\right\|^{2}
\end{aligned}
$$

In each process, the intensity difference between the transmitted signal and the received signal is compared. If the difference has the minimum value at the kth impedance, the impedance index becomes $\mathrm{k}$, and the index can be detected.

\section{SIMULATION RESULTS AND DISCUSSION}

In this section, the performance of the proposed scheme is evaluated by simulation. We assumed that the receiver knows the channel state information (CSI) perfectly in the Rayleigh fading channel. To evaluate the proposed scheme, M-ary phase shift keying (M-PSK) is used for the conventional modulation scheme [18], [19].

In Figure 5, we show the comparison of the spectral efficiency of the proposed system and M-PSK. The spectral efficiency is the amount of information that can be transmitted over a given bandwidth in a communication system [20]. The spectral efficiency is expressed as follows,

$$
R_{I I M}=\log _{2} M+\log _{2}\left\lfloor N_{Z}\right\rfloor
$$

Where $N_{Z}$ is the total number of impedances, $M$ is the modulation order. To verify the performance, we consider two impedances, named IIM $\left(N_{Z}=2\right)$, and 4 impedances, which is $\operatorname{IIM}\left(N_{Z}=4\right)$ with BPSK. To match same bpcu (bit per channel use), the conventional modulation is used QPSK for 2bpcu and 8-PSK for 3bpcu. When SNR is $18 \mathrm{~dB}$, both of the IIM $\left(N_{Z}=2\right)$ with BPSK and the convential modulation for QPSK can be reached spectral efficiency of $2 \mathrm{bps} / \mathrm{Hz}$. Also, both of the IIM $\left(N_{Z}=4\right)$ with BPSK and 8-PSK can be reached to $3 \mathrm{bps} / \mathrm{Hz}$. However, comparing in low SNR, the spectral efficiency of the proposed system show better performance than the conventional modulation techniques QPSK and 8-PSK in the same bpcu.

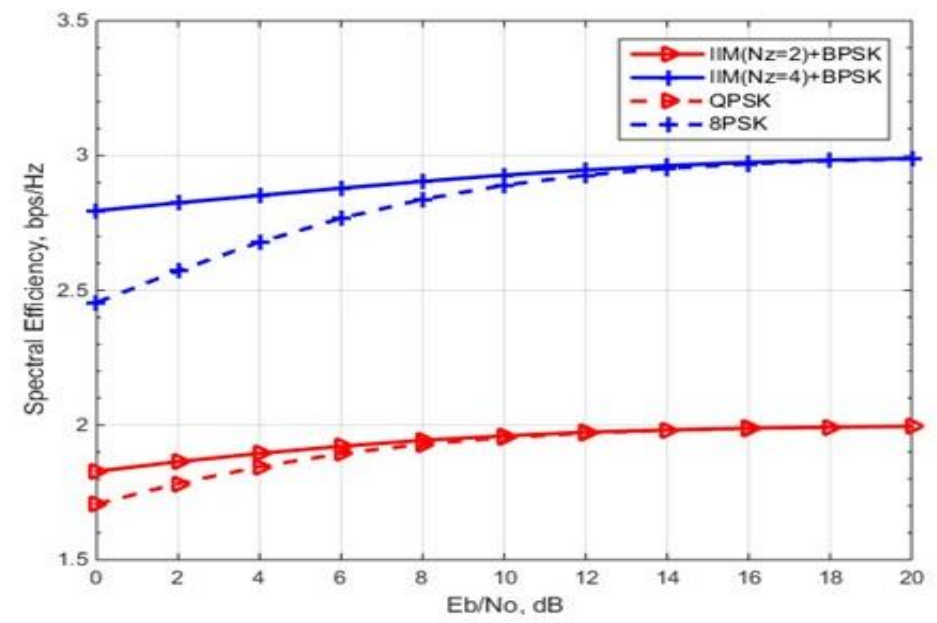

Figure 5. Spectral efficiency with different value of $N_{Z}$

In Figure 6, we present BER of our proposed system and the conventional modulation scheme according to the number of impedances. The parameter is equal to the fig. 5 e.g. the number of impedance and the modulation order. As a result, When BER is $10^{-2}$, it is improved by about $0.5 \mathrm{~dB}$ in case of $N_{Z}=2$, 
and about $1 \mathrm{~dB}$ in case of $N_{Z}=4$ compared with conventional modulation BER of IIM performance improved compared to M-PSK modulation. In addition, compared with different impedance number in IIM, when the number of impedance is increased, BER performance decreases in the simulation result. Especially, at low SNR, BER is better in the less number of impedance, i.e., the proposed technique is strong against noise.

Figure 7 shows the result of BER of the proposed modulation scheme at 4bpcu. The case of IIM $\left(N_{Z}=4\right)$ with QPSK are compared with 16PSK. At the BER is $10^{-2}$, the value of IIM is $17 \mathrm{~dB}$ and $16 \mathrm{PSK}$ is pointed $19 \mathrm{~dB}$, so the performance improved.

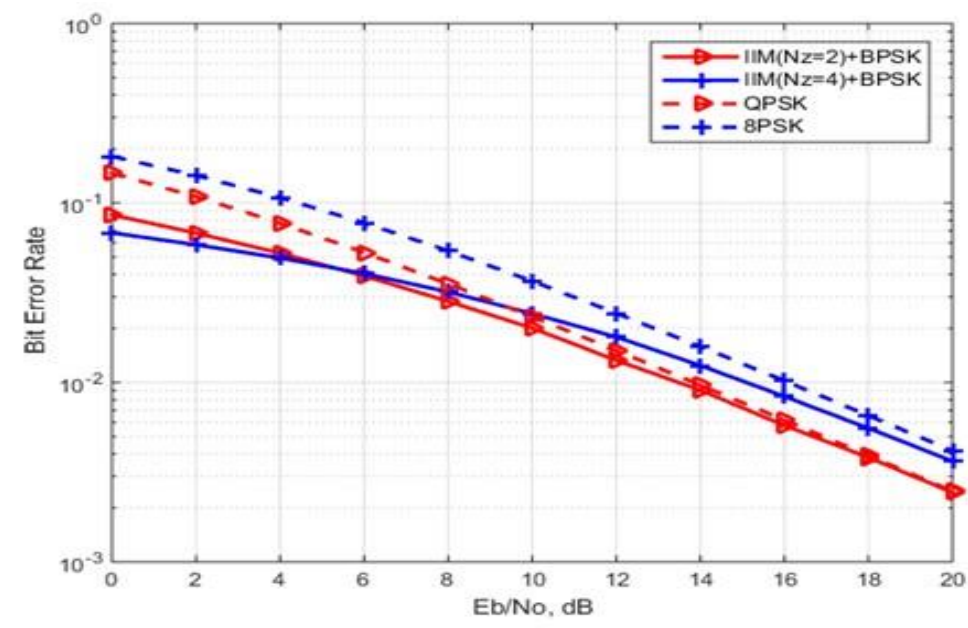

Figure 6. BER vs SNR of IIM and M-PSK

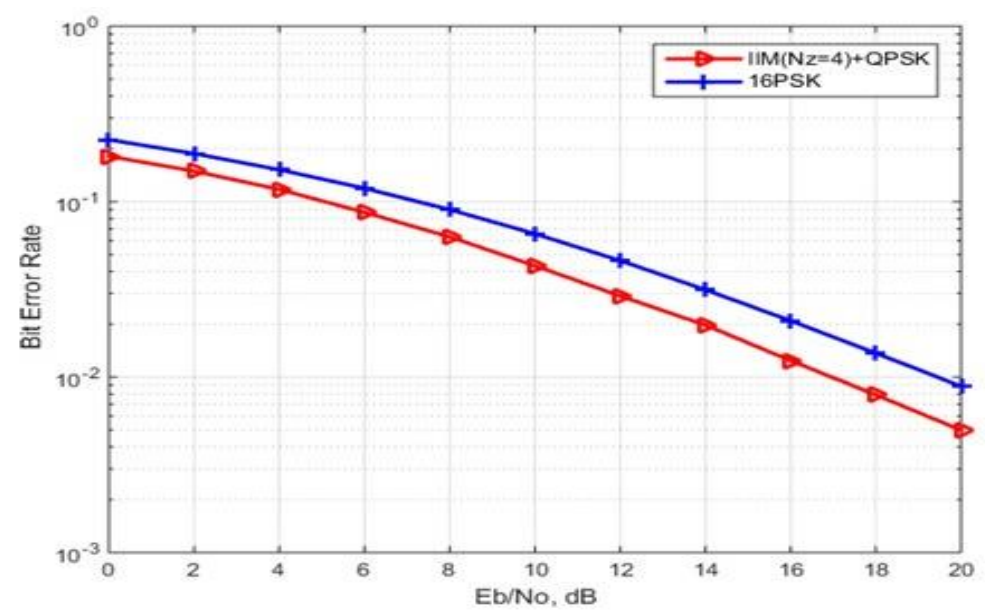

Figure 7. BER vs SNR of IIM and M-PSK in 4bpcu

\section{CONCLUSION}

In this paper, we suggest a novel modulation scheme using impedance matching, called IIM, to overcome explosive increaing of data traffic in $5 \mathrm{G}$ and beyond $5 \mathrm{G}$. The key principle of the proposed system is to give indices of the result that impedance match or mismatch between transiver. To detect impedance index, we present the detecting algorithm by deriving the maximum power transfer theorem. According to the simulation result, the impedance index modulation scheme improves both the spectral efficiency and BER compared to the traditional modulation scheme. For the future, we analyze the optimal impedance value for our proposed system. 


\section{ACKNOWLEDGEMENTS}

This work was supported by Priority Research Centers Program through the National Research Foundation of Korea (NRF) funded by the Ministry of Education, Science and Technology (2018R1A6A1A03024003).

\section{REFERENCES}

[1] Agiwal, Mamta, Abhishek Roy, and Navrati Saxena. "Next generation 5G wireless networks: A comprehensive survey." IEEE Communications Surveys \& Tutorials 18.3 (2016): 1617-1655.

[2] Cisco, Visual Networking Index. "Global Mobile Data Traffic Forecast Update, 2015-2020 White Paper." Document ID958959758 (2016).

[3] Wang, Cheng-Xiang, et al. "Cellular architecture and key technologies for 5G wireless communication networks." IEEE Communications Magazine 52.2 (2014): 122-130.

[4] Van Trees, Harry L. Detection, estimation, and modulation theory, part I: detection, estimation, and linear modulation theory. John Wiley \& Sons, 2004.

[5] Xuehong, Sun, et al. "A 3-D Polarization Quadrature Amplitude Modulation method and constellation mapping." China Communications 12.10 (2015): 16-26.

[6] Basar, Ertugrul, et al. "Index modulation techniques for next-generation wireless networks." IEEE Access 5 (2017): 16693-16746.

[7] Basar, Ertugrul. "Index modulation techniques for 5G wireless networks." IEEE Communications Magazine 54.7 (2016): 168-175.

[8] Mesleh, Read, et al. "Spatial modulation-a new low complexity spectral efficiency enhancing technique." Communications and Networking in China, 2006. ChinaCom'06. First International Conference on. IEEE, 2006.

[9] Abu-Alhiga, Rami, and Harald Haas. "Subcarrier-index modulation OFDM." Personal, Indoor and Mobile Radio Communications, 2009 IEEE 20th International Symposium on. IEEE, 2009.

[10] Di Renzo, Marco, et al. "Spatial modulation for generalized MIMO: Challenges, opportunities, and implementation." Proceedings of the IEEE 102.1 (2014): 56-103.

[11] Mesleh, Raed, et al. "Spatial modulation." IEEE Transactions on Vehicular Technology 57.4 (2008): 2228.

[12] Ganesan, Sudharsan, et al. "On the performance of spatial modulation OFDM." Signals, Systems and Computers, 2006. ACSSC'06. Fortieth Asilomar Conference on. IEEE, (2006).1825-1829.

[13] Hasan, Rawaa J., and Hikmat N. Abdullah. "Comparative study of selected subcarrier index modulation OFDM schemes." TELKOMNIKA (Telecommunication Computing Electronics and Control) 17.1 (2019).

[14] Xiao, Yue, et al. "OFDM with interleaved subcarrier-index modulation." IEEE Communications Letters 18.8 (2014): 1447-1450.

[15] Shen, Shanpu, and Ross D. Murch. "Impedance matching for compact multiple antenna systems in random RF fields." IEEE Transactions on Antennas and Propagation 64.2 (2016): 820-825.

[16] I. Adam, M.N. M. Yasin, et al. "Rectifier for RF energy harvesting using stub matching" Indonesian Journal of Electrical Engineering and Computer Science 13.3 (2019).

[17] Mohammed Lahsaini, Lahbib Zenkouar, et al. "Modeling of a Microwave Amplifier Operating around 11 GHz for Radar Applications" Indonesian Journal of Electrical Engineering and Computer Science 8.5(2018): 3496-3503.

[18] De Mingo, Jesus, et al. "An RF electronically controlled impedance tuning network design and its application to an antenna input impedance automatic matching system." IEEE Transactions on Microwave Theory and Techniques 52.2 (2004): 489-497.

[19] Kim, Jaeseok. Automated matching control system using load estimation and microwave characterization. University of Florida, 2008

[20] Sharma, Vineet, et al. "BER performance of OFDM-BPSK,-QPSK,-QAM over AWGN channel using forward Error correcting code." International Journal of Engineering Research and Applications (IJERA) 2.3 (2012): 1619-1624.

[21] Rao, K. Deergha. Channel coding techniques for wireless communications. Springer India, 2015.

[22] Foschini, Gerard J., et al. "Simplified processing for high spectral efficiency wireless communication employing multi-element arrays." IEEE Journal on Selected areas in communications 17.11 (1999): 1841-1852. 